\title{
BMP9/p38 MAPK is essential for the antiproliferative effect of resveratrol on human colon cancer
}

\author{
SHUANG-XUE YUAN ${ }^{1,2}$, DONG-XU WANG ${ }^{1,2}$, QIU-XIANG WU ${ }^{1,2}$, CHUN-MEI REN $^{1,2}$, YANG LI ${ }^{1,2}$, \\ QIAN-ZHAO $\mathrm{CHEN}^{1,2}$, YU-HUA ZENG ${ }^{1,2}$, YING SHAO $^{1,2}$, JUN-QIN YANG $^{1,2}$, YAN BAI ${ }^{1,2}$, \\ PU ZHANG ${ }^{1,2}$, YU YU ${ }^{1,3}$, KE WU ${ }^{1,2}$, WEN-JUAN SUN ${ }^{1,2}$ and BAI-CHENG HE ${ }^{1,2}$ \\ ${ }^{1}$ Chongqing Municipal Key Laboratory of Higher Education Institutions for Biochemistry and Molecular Pharmacology; \\ Departments of ${ }^{2}$ Pharmacology and ${ }^{3}$ Chemistry, School of Pharmacy, Chongqing Medical University, \\ Yuzhong, Chongqing, P.R. China
}

Received August 13, 2015; Accepted October 12, 2015

DOI: $10.3892 /$ or.2015.4407

\begin{abstract}
Colon cancer is one of the most common malignancies of the digestive system. Although more effective therapeutic strategies have been developed in the last decades, there is still a great clinical need to explore new treatment regimens for colon cancer due to the undesirable prognosis. In the present study, we investigated the anticancer activity of resveratrol (Res) in human colon cancer cells, and the possible mechanism underlying this effect. We employed crystal violet staining, flow cytometry and western blotting to test the antiproliferation- and apoptosis-inducing effects of Res in LoVo cells. A xenograft tumor model was also introduced to confirm the in vivo anticancer effect of Res. Using PCR, western blotting, a recombinant adenovirus and a specific inhibitor of p38 MAPK or bone morphogenetic protein receptor (BMPR) to explore the possible molecular mechanisms. We found that Res markedly inhibited the proliferation and promoted the apoptosis of LoVo cells, and suppressed the in vivo tumor growth of colon cancer. Res substantially upregulated the expression of bone morphogenetic protein 9 (BMP9). Exogenous expression of BMP9 enhanced the anticancer effect of Res in LoVo cells, while BMP9 knockdown partly reduced this activity. Res increased the activation of p38 MAPK, which was enhanced by the exogenous expression of BMP9. The anticancer activity of Res, or Res combined with BMP9, was reduced partly by the p38 MAPK inhibitor. The BMPR inhibitor almost abolished the Res-induced activation of p38 MAPK, and attenuated the antiproliferative effect of Res in the LoVo cells. Our findings strongly suggest that the anticancer effect of Res in human colon cancer cells may be partly mediated by upregulation of BMP9 to activate p38 MAPK in a BMPR-dependent manner.
\end{abstract}

Correspondence to: Professor Bai-Cheng He, Department of Pharmacology, School of Pharmacy, Chongqing Medical University, 1 Yixueyuan Road, Yuzhong, Chongqing 400016, P.R. China

E-mail: 894704897@qq.com; hebaicheng99@yahoo.com

Key words: resveratrol, colon cancer, anticancer, BMP9, p38 MAPK

\section{Introduction}

Colon cancer is one of the most common malignancies in the digestive system. Current treatments include surgical resection, radiotherapy, chemotherapy and targeted therapy alone or in combination. However, the prognosis remains unsatisfactory $(1,2)$. Thus, there is a great clinical need to explore new agents for the treatment of colon cancer.

Numerous herbs or their components have been clinically used as potential candidates for anticancer agents, such as camptothecin, vincristine and taxol (3-5). Polyphenolic compounds are highly present in red wine, and some reports and clinical epidemiologic studies have demonstrated that red wine contributes to a reduction in cardiovascular diseases and cancer risk (6,7). Resveratrol (3,5,4'-trihydroxystilbene, Res), a polyphenolic compound from beans and grapes, was discovered in red wine by Siemann and Creasy in 1992, and can be used as a platelet aggregation inhibitor, cardiac-protection and anticancer agent $(8,9)$. It has been reported that Res can inhibit the proliferation and promote the apoptosis of various types of cancer cells, such as colon, breast and prostate (10-12). For colon cancer, although the anticancer effects of Res have been validated (10), the precise mechanisms underlying these activities remain unclear.

Bone morphogenetic proteins (BMPs) belong to the transforming growth factor- $\beta$ (TGF- $\beta$ ) superfamily (13). Approximately 20 BMPs have been identified to date, and they exert multifunctions to regulate physiological processes, such as proliferation, differentiation, adhesion, migration and apoptosis $(14,15)$. Thus, the aberrant expression of BMPs and/or downstream signal cascades have been implicated in the pathogenesis of various types of cancer, such as colon cancer $(16,17)$. Therefore, BMPs and/or the downstream signal cascades may be used as potential targets for colon cancer treatment $(15,18)$. BMP9 is the most potent BMP member to induce osteogenic differentiation in mesenchymal stem cells to date (19). Apart from promoting osteogenic differentiation, BMP9 is also implicated in cancer, although the outcome is controversial. BMP9 inhibits the proliferation of breast and prostate cancer cells $(20,21)$, but promotes the proliferation of osteosarcoma, ovarian and liver cancer cells (22-24). The 
response of cancer cells to BMP9 may greatly differ even in the same type of cancer by inhibiting or promoting proliferation, such as in osteosarcoma $(22,25)$. BMP9 often onsets its signaling through the canonical BMP/Smad pathway. Namely, BMP9 binds with type II bone morphogenetic protein receptor (BMPR) or type I BMPR, then phosphorylates Smad1/5/8 and forms a complex with Smad4, followed by translocation to the nucleus and regulation of downstream targets $(19,26)$. In addition, BMP9 can also exert its function through the non-canonical BMP/Smad pathway, such as p38 MAPK and PI3K/Akt $(26,27)$. The p38 MAPK signal is involved in cell differentiation, apoptosis and autophagy (28). Activation of p38 MAPK is critical for BMP9 to induce osteogenic differentiation in mesenchymal stem cells, but the relationship between p38 MAPK and BMP9 in cancer is not yet known.

Although the anticancer effect of Res in colon cancer cells has been well validated, it remains unknown whether BMP9 is associated with this activity. In the present study, we investigated the possible role of BMP9 in the antiproliferative effect of Res on LoVo cells, and we elucidated how BMP9 exerts this function. Our findings demonstrated that Res can effectively inhibit the proliferation and promote the apoptosis of LoVo cells, which may be partly mediated by upregulation of BMP9 to activate 38 MAPK. Hence, Res may be used as an effective anticancer agent for colon cancer treatment alone or in combination with other agents, and BMP9/p38 MAPK signaling may be a potential therapeutic target for colon cancer treatment.

\section{Materials and methods}

Reagents and cell culture. Res was purchased from Xi'an Hao-xuan Biotechnology Co. Ltd. (Xi'an, China). For the in vivo experiments, Res was prepared with $0.5 \%$ carboxymethylcellulose sodium (CMC-Na) as a suspension. The LoVo cell line was obtained from the American Type Culture Collection (ATCC; Manassas, VA, USA). All antibodies were purchased from Santa Cruz Biotechnology (Santa Cruz, CA, USA). LDN-193189 and SB203580 were purchased from Selleckchem (Houston, TX, USA). Cells were maintained in Dulbecco's modified Eagle's medium (DMEM) with $10 \%$ fetal bovine serum (FBS), penicillin $(100 \mathrm{U} / \mathrm{ml})$ and streptomycin $(100 \mu \mathrm{g} / \mathrm{ml})$ at $37^{\circ} \mathrm{C}$ in $5 \% \mathrm{CO}_{2}$.

Crystal violet assay. The crystal violet assay was carried out as previously described (10). Experimentally, LoVo cells were treated with different concentrations of Res. Cells were carefully washed at the scheduled time points with cold phosphate-buffered saline (PBS; $4^{\circ} \mathrm{C}$ ), and were stained with $0.5 \%$ crystal violet solution for $20-30 \mathrm{~min}$ (at room temperature). Plates were gently washed with tap water, air dried at room temperature, then scanned and quantified. For quantification, $20 \%$ acetic acid was used to extract the crystal violet for $20 \mathrm{~min}$, with gentle shaking at room temperature. The absorbance at $570 \mathrm{~nm}$ was measured.

Flow cytometric analysis of cell cycle distribution and apoptosis. Cells were seeded into 6-well plates. For cell cycle analysis, the cells were treated with different concentrations of Res or DMSO. After $48 \mathrm{~h}$, the cells were washed with cold PBS $\left(4^{\circ} \mathrm{C}\right)$, collected and washed with cold $\left(4^{\circ} \mathrm{C}\right) 70 \%$ ethanol followed by washing with 50 and $30 \%$ ethanol, and PBS. Then, the cells were incubated with $1 \mathrm{ml}$ of $20 \mathrm{mg} / \mathrm{ml}$ propidium iodide (PI) containing RNase $(1 \mathrm{mg} / \mathrm{ml})$ for $30 \mathrm{~min}$ (in PBS), followed by fluorescence activated cell sorting (FACS) analysis. For the apoptosis analysis, the cells were collected after treatment with the different concentrations of Res or DMSO for $48 \mathrm{~h}$. Then, the cells were washed with cold PBS, incubated with Annexin V-EGFP and PI according to the procedure provided in the kit (\#KGA104; KeyGen Biotech, China). Finally, the cells were subjected to FACS assay.

Western blot assay. Cells were seeded into 6-well plates, and were treated with different concentrations of Res or DMSO. At the scheduled time points, the cells were lysed and the lysates were boiled for $10 \mathrm{~min}$. Total protein was separated with SDS-PAGE, transfered to polyvinylidene difluoride (PVDF) membranes, blocked with 5\% BSA and probed with the antibody tagged with HRP. The target bands were developed with ECL substrate (\#34095; Thermo, USA).

Total RNA extraction and reverse transcription polymerase chain reaction $(R T-P C R)$ assay. The cells were seeded in a T25 flask and were treated with different concentrations of Res or DMSO. Total RNA was extracted with TRIzol reagent (Invitrogen, Carlsbad, CA, USA), followed by RT reaction to obtain cDNA. Finally, the cDNA products were used as templates to detect the expression of the target genes with PCR. Primer sequences are available upon request.

Construction of recombinant adenoviruses for exogenous expression of BMP9, GFP and small interference RNA fragments for BMP9. The recombinant adenoviruses for exogenous expression of BMP9 (AdBMP9) or GFP (AdGFP) were generated following the AdEasy system as previously reported $(29,30)$, as well as the recombinant adenoviruses expressing small interfering RNA (siRNA) fragments for BMP9 (AdsiBMP9). AdGFP was used as a vehicle control.

Ectopic tumor model of human colon cancer and histological evaluation. All experiments followed the guideline of the Institutional Animal Care and Use Committee of Chongqing Medical University (Chongqing, China). Athymic nude mice (female, 4-6 weeks old, 5/group) were purchased from the Animal Center of Chongqing Medical University (Chongqing, China). LoVo cells were cultured and resuspended in PBS $\left(4^{\circ} \mathrm{C}\right)$ for implantation into the flanks of the athymic nude mice as previously reported $(10,31)$. The mice were treated with Res ( 50 or $150 \mathrm{mg} / \mathrm{kg}$ ) or the same volume of solvent through intragastric administration 1 week after implantation, once a day, up to 4 weeks. At the end of the 4 week, all nude mice were sacrificed. The tumor samples were harvested, fixed in $10 \%$ formalin and embedded in paraffin. Sections of the samples were subjected to hematoxylin and eosin (H\&E) staining.

Immunohistochemical staining. The deparaffinized slides were subjected to antigen retrieval and were probed with an anti-BMP9 antibody or isotype IgG control, followed by incubation with a biotin secondary antibody and streptavidin-HRP. The proteins of interest were visualized by 3,3'-diaminobenzidine staining. 
A
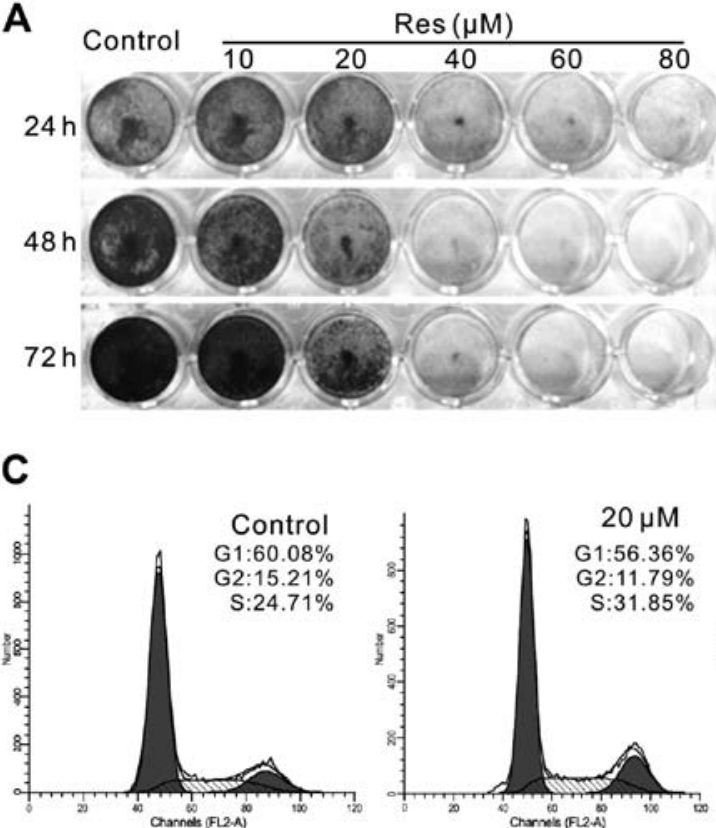

B

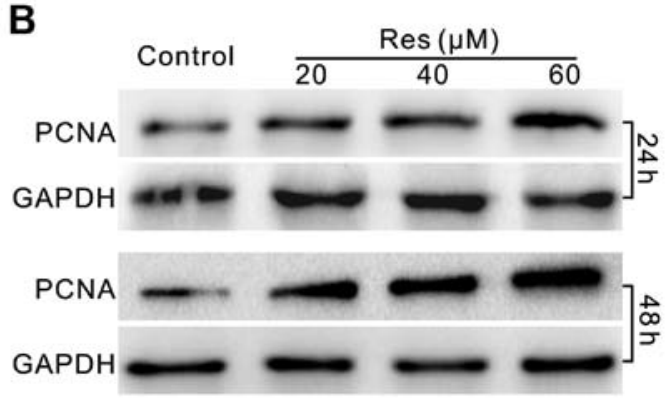

Figure 1. Resveratrol (Res) inhibits the proliferation of LoVo cells. (A) Crystal violet staining results show the antiproliferation effect of Res on the LoVo cells. (B) Western blot results show the effect of Res on the expression of PCNA in LoVo cells. GAPDH was used as a loading control. (C) Cell cycle analysis shows the $\mathrm{S}$ phase arrest effect of Res in the LoVo cells.

A

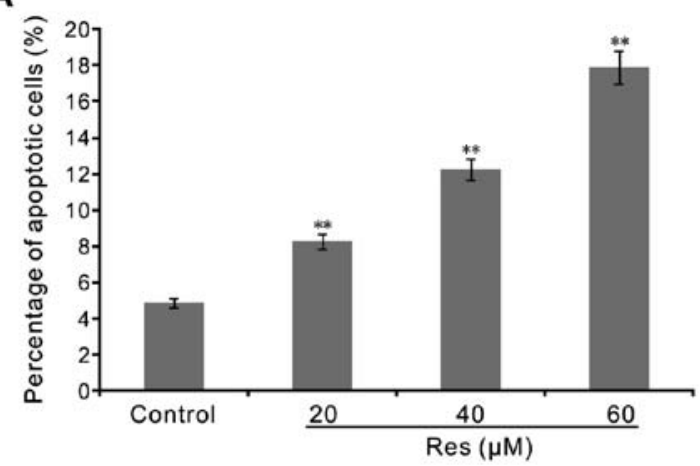

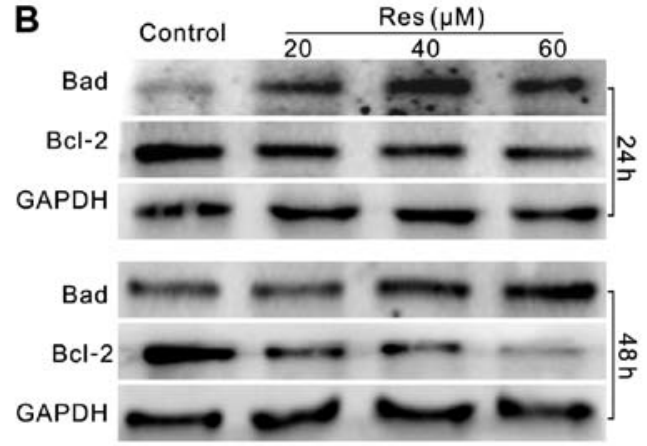

Figure 2. Resveratrol (Res) induces apoptosis in LoVo cells. (A) FACS assay results show the percentage of apoptotic cells induced by Res ("*w $<<0.01$ vs. the control group). (B) Western blot results show the effect of Res on the Bad and Bcl-2 levels in the LoVo cells. GAPDH was used as a loading control.

Statistical analysis. All experiments were performed in triplicates and the results were repeated in at least three independent experiments. Statistical analysis of the results was conducted following a t-test (Microsoft Excel). Data are expressed as mean \pm standard deviation (SD).

\section{Results}

Res decreases the proliferative ability of LoVo cells. It has been reported that Res exhibits antiproliferative activity in various types of cancer cells, including colon cancer cells. In this investigation, we first confirmed the effect of Res on LoVo cells. The results showed that Res inhibited the proliferation of LoVo cells in a concentration-dependent manner (Fig. 1A). The level of proliferating cell nuclear antigen (PCNA) was also markedly increased in a concentration-dependent manner (Fig. 1B). Cell cycle analysis results showed that Res induced cell cycle arrest at the S phase in the LoVo cells (Fig. 1C). All these data suggest that Res inhibits the proliferation of LoVo cells.

Res induces the apoptosis of LoVo cells. Most anticancer agents induce apoptosis, therefore we ascertained whether Res induces apoptosis in the LoVo cells. We employed FACS and western blotting to analyze the effect of Res on apoptosis in the LoVo cells. FACS assay results showed that Res notably increased the percentage of apoptotic LoVo cells (Fig. 2A). Western blot assay showed that Res increased the level of Bad, but markedly decreased the level of Bcl-2 prominently in a concentration-dependent manner (Fig. 2B). These results confirmed that Res may be an effective apoptosis inducer in human colon cancer cells.

Res inhibits tumor growth in an ectopic tumor model. The above findings showed that Res is a potent proliferation 

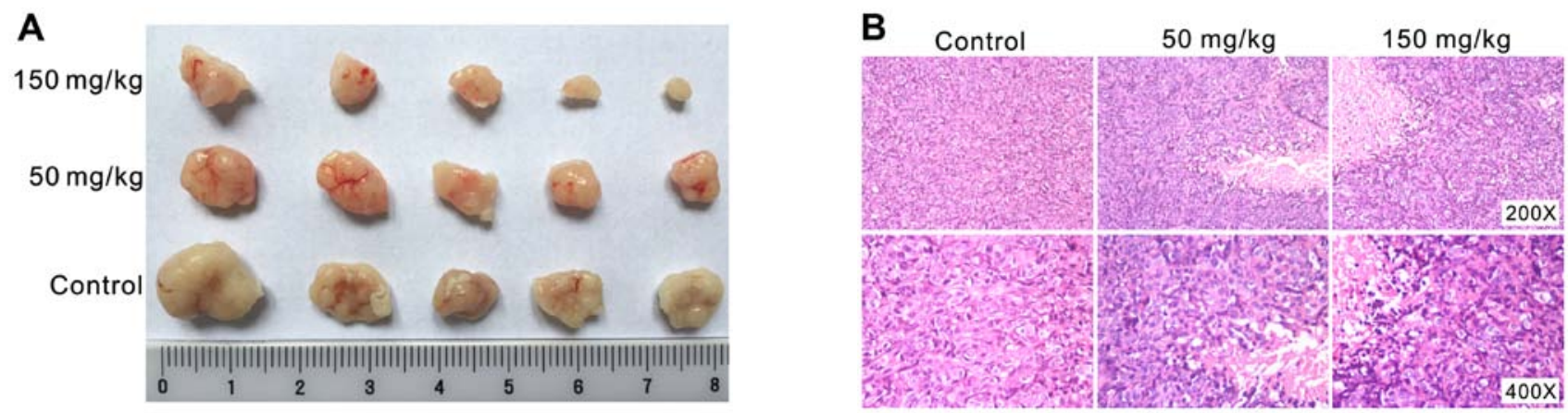

Figure 3. Resveratrol (Res) inhibits the growth of tumors. (A) Representative tumor masses from the xenograft tumor assay. (B) Hematoxylin and eosin staining results show the antitumor activity of Res in colon cancer. Representative images are shown.
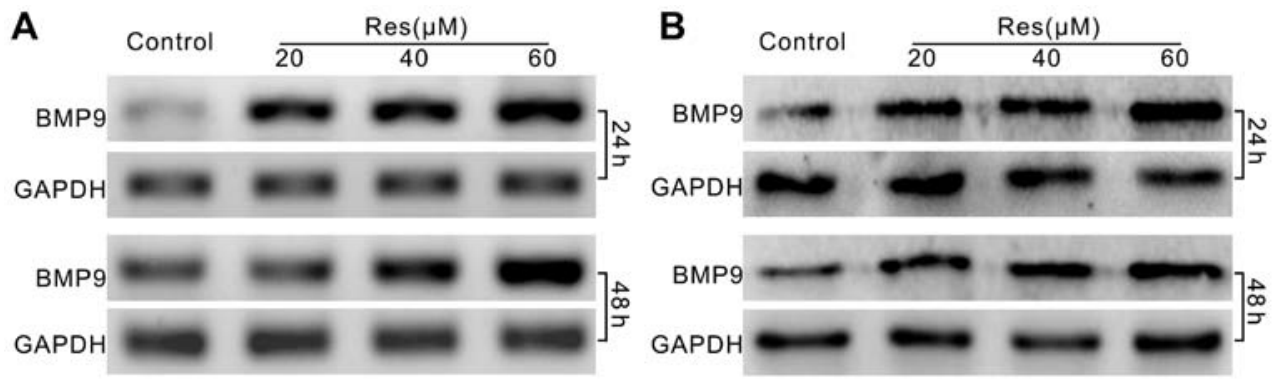

C
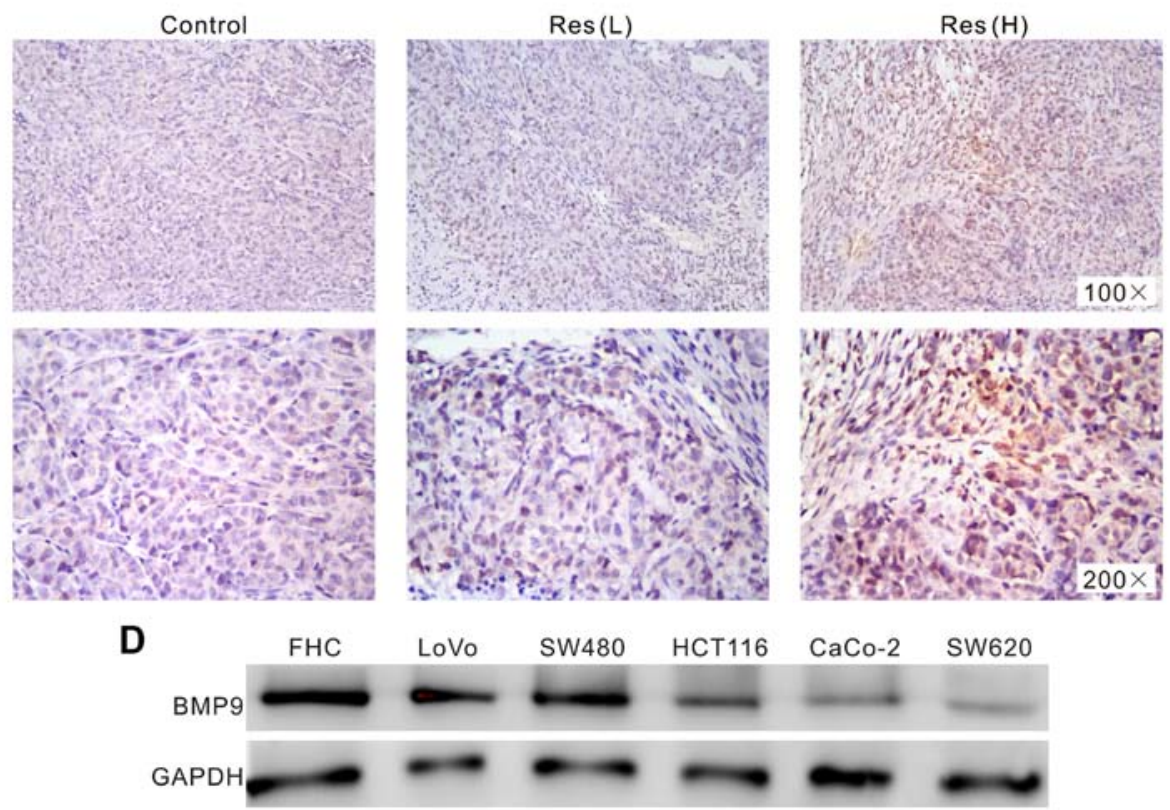

Figure 4. Resveratrol (Res) increases BMP9 expression in the LoVo cells. (A) PCR assay results show the effect of Res on BMP9. GAPDH was used as a loading control. (B) Western blot analysis shows the effect of Res on BMP9. GAPDH was used as a loading control. (C) Immunohistochemical staining results show the expression of BMP9 (L, low dosage, $50 \mathrm{mg} / \mathrm{kg} ; \mathrm{H}$, high dosage, $150 \mathrm{mg} / \mathrm{kg}$ ). (D) Western blot assay shows the endogenous expression of BMP9 in FHC cells and in different colon cancer cell lines. GAPDH was used as a loading control.

inhibitor in colon cancer cells. We next investigated the in vivo anticancer activity of Res with a well-established xenograft tumor model as previously reported $(10,31)$. We implanted $1 \times 10^{6} \mathrm{LoVo}$ cells into the flanks of athymic nude mice. One week after implantation, the mice were treated with Res (50 or $150 \mathrm{mg} / \mathrm{kg}$ ) through intragastric administration, once a day, up to 4 weeks. The results showed that tumor masses from the Res-treated groups were smaller than those from the control group (Fig. 3A). H\&E staining results showed that cellularity was apparently decreased in the Res-treated groups (Fig. 3B). These results demonstrated that Res is capable of effectively inhibiting colon cancer growth in vivo.

Res increases the expression of BMP9 in LoVo cells. Next, we aimed to ascertain the possible mechanism underlying the anticancer activity of Res in the colon cancer cells. Using PCR and western blot assay we found that BMP9 was highly upregulated by Res in the LoVo cells (Fig. 4A and B). The 
A
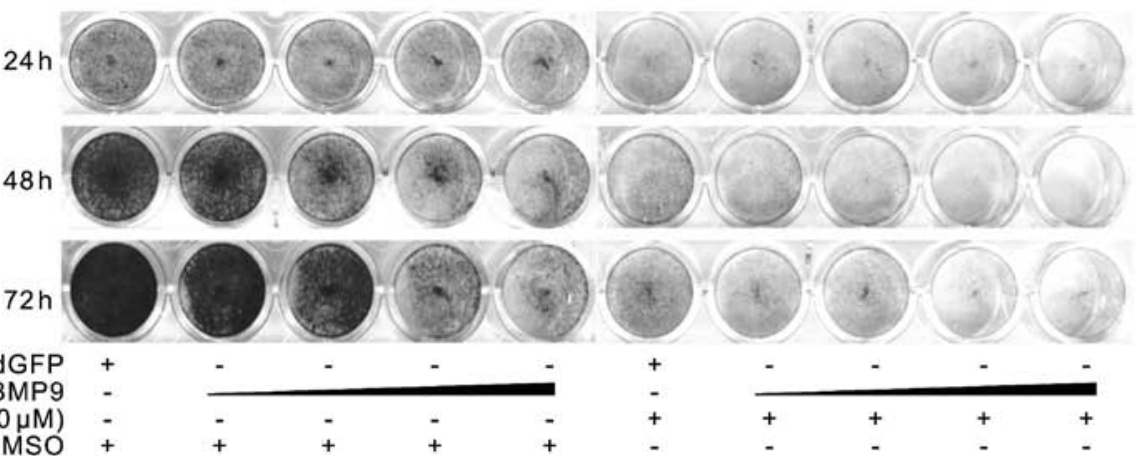

B $48 h$
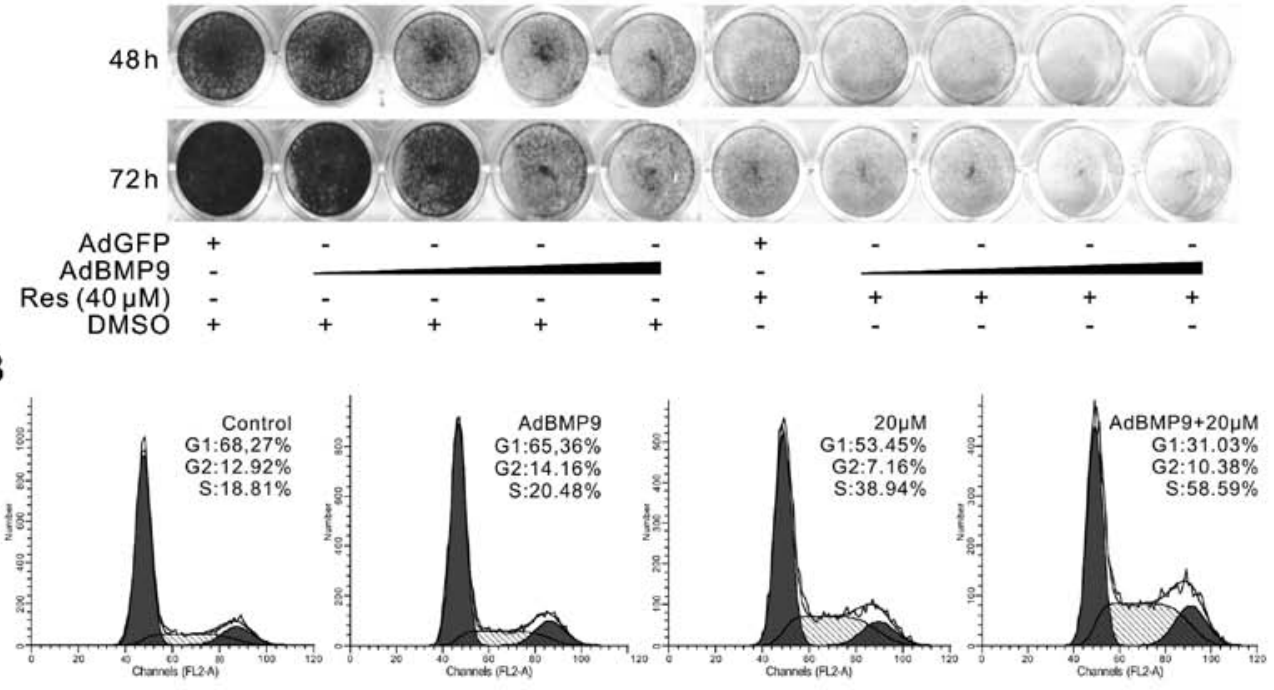

C $24 \mathrm{~h}$
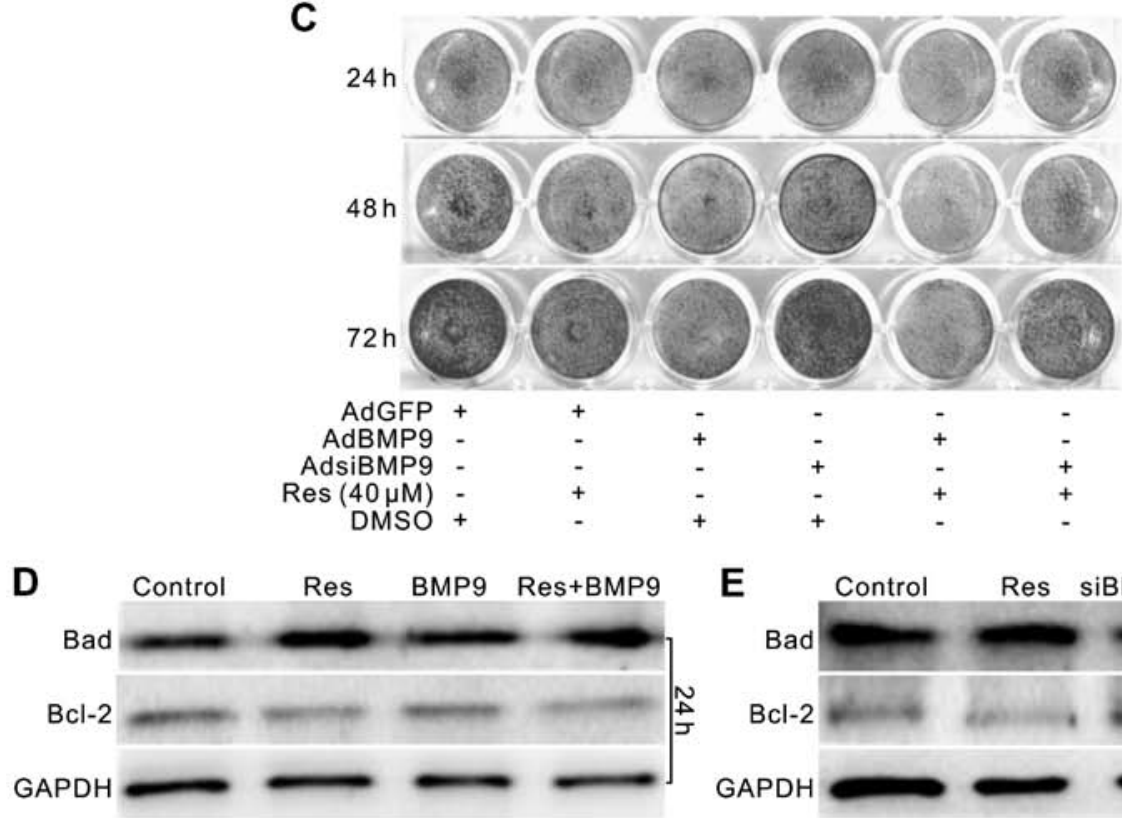

E
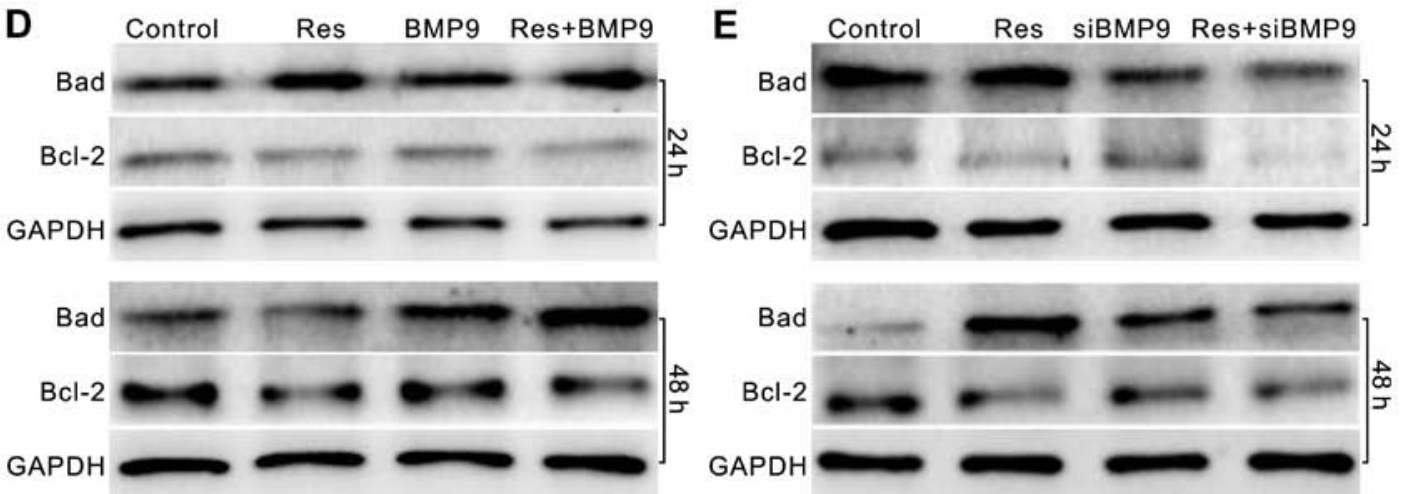

Figure 5. BMP9 affects the anticancer effect of resveratrol (Res) in the LoVo cells. (A) Crystal violet staining results show the effect of exogenous expression of BMP9 on the antiproliferative effect of Res on the LoVo cells. (B) Cell cycle analysis results show the effect of exogenous expression of BMP9 on the cell cycle arrest induced by Res in LoVo cells. (C) Crystal violet staining results show the effect of exogenous expression or knockdown of BMP9 on the antiproliferative effect of Res on the LoVo cells. (D) Western blot assay shows the effect of exogenous expression of BMP9 on Bad and Bcl-2 levels in the LoVo cells. GAPDH was used as a loading control. (E) Western blot assay show the effect of BMP9 knockdown on Bad and Bcl-2 levels in the LoVo cells. GAPDH was used as a loading control.

immunohistochemical staining of the tumor masses showed similar results (Fig. 4C). Furthermore, western blot assay found that BMP9 was detectable in all the colon cancer cell lines, as well as FHC cells. However, the level of BMP9 in the FHC cells was relative higher than that in the cancer cell lines (Fig. 4D). These data imply that the upregulation of
BMP9 may be related with the anticancer activity of Res in colon cancer.

BMP9 enhances the antiproliferative activity of Res in LoVo cells. As Res upregulates the expression of BMP9 in LoVo cells and the level of BMP9 is higher in FHC cells, we aimed 

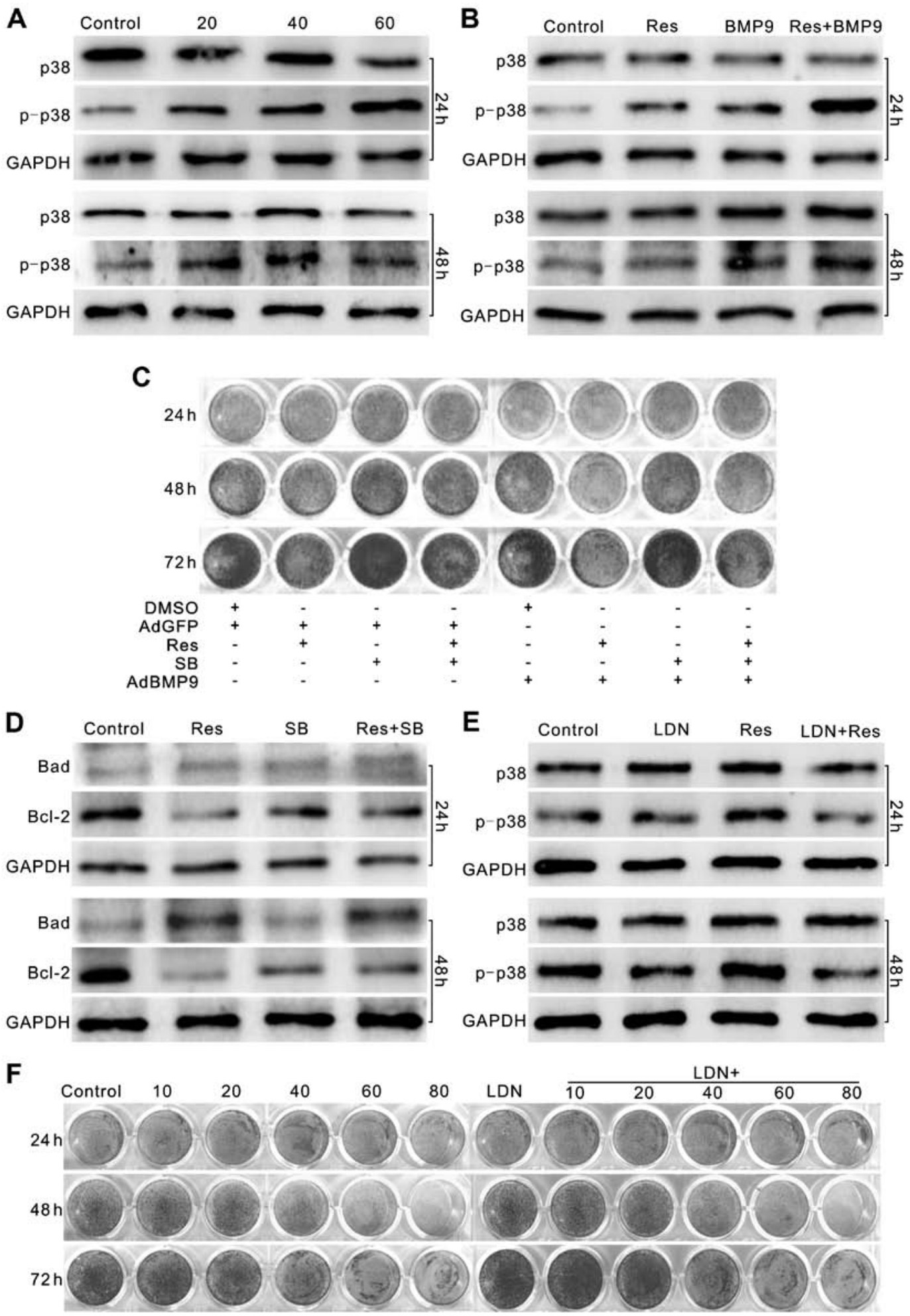

Figure 6. BMP9 activates p38 MAPK to promote the anticancer activity of Res in LoVo cells. (A) Western blot assay shows the effect of Res on the activation of p38 MAPK in the LoVo cells. GAPDH was used as a loading control. (B) Western blot assay shows the effect of Res and/or exogenous expression of BMP9 on the activation of p38 MAPK in the LoVo cells. GAPDH was used as a loading control. (C) Crystal violet staining results show the effect of BMP9 and/or p38 inhibitor on the antiproliferation effect of Res in the LoVo cells (SB, p38 inhibitor SB203580). (D) Western blot assay shows the effect of the p38 inhibitor and/or Res on Bad and Bcl-2 levels in the LoVo cells (SB, p38 inhibitor SB203580). GAPDH was used as a loading control. (E) Western blot assay shows the effect of the BMPR inhibitor and/or Res on the activation of p38 MAPK in the LoVo cells (LDN, BMPR inhibitor LDN-193189). GAPDH was used as a loading control. (F) Crystal violet staining results show the effect of Res and/or the BMPR inhibitor on the proliferation in the LoVo cells (LDN, BMPR inhibitor LDN-193189).

to ascertain whether BMP9 affects the anticancer activity of Res in LoVo cells. Using the AdEasy system, we constructed recombinant adenoviruses to express BMP9 or BMP9 siRNA fragments in the LoVo cells. Using a crystal violet staining assay, we found that exogenous expression of BMP9 inhibited the proliferation of LoVo cells, and the antiproliferative 
effect of Res on LoVo cells was enhanced when combined with BMP9 (Fig. 5A). Cell cycle analysis showed that BMP9 apparently enhanced the $S$ phase arrest effect of Res in LoVo cells (Fig. 5B). BMP9 knockdown partly reversed the antiproliferative effect of Res (Fig. 5C). Western blot assay showed that BMP9 increased the level of Bad but decreased the level of Bcl-2 induced by Res in the LoVo cells (Fig. 5D). However, knockdown of BMP9 decreased the level of Bad induced by Res in the LoVo cells, although no apparent effect was noted for Bcl-2 (Fig. 5E). These data indicate that the anticancer effect of Res in colon cancer may be mediated by upregulating BMP9.

p38 MAPK mediates the effect of BMP9 on the antiproliferative activity of Res in LoVo cells. BMP9 usually exerts its function through the canonical BMP/Smad pathway, but our findings showed that Res exhibited no apparent effect on the phosphorylation of Smad1/5/8 (data not shown). This implied that the effect of BMP9 on the antiproliferative effect of Res noted in the LoVo cells may not be mediated through the canonical BMP/Smad pathway, but the non-canonical BMP/Smad pathway. p38 MAPK is involved in this signaling pathway, and its activation is essential for BMP9-induced osteogenesis in mesenchymal stem cells (27). Therefore, we speculated that Res-induced BMP9 may affect the activation of p38 MAPK in LoVo cells. Western blot results showed that Res increased the level of phosphorylated p38 MAPK (p-p38) substantially in the LoVo cells (Fig. 6A), and exogenous expression of BMP9 enhanced this effect of Res on p-p38 (Fig. 6B). The p38 MAPK inhibitor, SB203580 (32), increased the proliferation of LoVo cells, while reducing the antiproliferative effect of Res, BMP9 and Res combined with BMP9 (Fig. 6C). The p38 MAPK inhibitor also reversed the Res-induced decrease in Bcl-2, although no apparent effect on the level of Bad was noted (Fig. 6D). The BMPR inhibitor (LDN-193189) abolished the Res-induced activation of p38 MAPK in the LoVo cells (Fig. 6E). Similar results were also found when BMP9 was knocked down (data not shown). Further analysis showed that the BMPR inhibitor promoted the proliferation of LoVo cells, and partly reversed the antiproliferative effect of Res on LoVo cells (Fig. 6F). These data suggest that BMP9 may mediate the antiproliferative effect of Res on LoVo cells by activating p38 MAPK, which may be partly triggered through a BMPR-dependent manner.

\section{Discussion}

Colon cancer, is the most common prevalent malignancy in the digestive system and accounts for a major proportion of cancer-induced mortality (33). Hence, there is a great clinical need to explore new agents or adjuvant therapies for colon cancer treatment. In the present study, we demonstrated the efficacious anticancer activity of Res in human colon cancer cells. Mechanistically, we found that the anticancer effect of Res on colon cancer cells may be mediated by activating p38 MAPK partly through upregulation of BMP9.

Challenges for colon cancer treatment include the toxicity of drugs, drug resistance and cancer cell metastasis. The serious side-effects associated with traditional chemotherapy drugs greatly decrease the life quality of patients, while reducing the effectiveness of the treatment for colon cancer $(34,35)$. Although targeted therapy agents, such as the VEGF antibody bevacizumab, and EGFR antibodies cetuximab and panitumumab $(2,36)$, have been clinically used, natural products and/or their derivates are still an essential source of anticancer agents (37). Resveratrol (Res), a natural polyphenolic compound, is found in the skin of red grapes or other fruits (8). Increasing evidence suggests that Res shows antiproliferationand apoptosis-inducing activities in breast, prostate and colon cancer cells (10-12). Since the bioavailability of Res is low, even a high dose of Res may not provide the effective concentration required for systemic treatment (38). However, Res may benefit gastrointestinal cancer treatment. Reports and our studies have validated the inhibitory effect of Res on the proliferation of colon cancer cells. Vanamala et al reported that Res induces apoptosis through suppressing $\mathrm{Wnt} / \beta$-catenin and activating the p53 signaling pathways in human colon cancer cells (39). Sheth et al found that microRNA-21 participates in the inhibition of prostate cancer cell growth and metastasis initialized by Res (40). Recently, it was reported that the p38 MAPK and PI3K signaling pathways are also involved in the anticancer activity of Res $(41,42)$. However, to date, the detail molecular mechanism underlying this process remains unclear.

BMP9, also termed growth and differentiation factor 2 (GDF2), was first identified in the developing mouse liver as playing an important role in regulating iron metabolism and the development of cholinergic neurons (43). Although BMP9 has been reported as the most potent BMP member to induce osteogenic differentiation in mesenchymal stem cells, it has also been implicated with tumorigenesis (20-25). BMP9 exerts its physiological function through the canonical BMP/Smad pathway or the non-canonical BMP/Smad pathway. Regarding the canonical BMP/Smad pathway, BMPs bind with BMP receptor (type I or type II) and phosphorylate BMP-related Smads (Smad1/5/8), form a complex with Smad4 and then translocate to the nucleus regulating downstream targets $(19,26)$. Regarding the noncanonical BMP/Smad pathway, BMPs activate a serial signaling pathway, such as PI3K/Akt and p38 MAPK $(27,44)$. However, how BMP9 activates these signaling pathways remains unknown. The response of cancer cells to BMP9 may depend on the cell type and/or the microenvironment of the cells. A few reports have indicated that BMP9 is important for hepatocellular carcinoma cell proliferation and survival, and promotes the proliferation of osteosarcoma and ovarian cancer cells $(22,23)$. On the contrary, it has also been reported that BMP9 can inhibit the proliferation or metastasis in various types of cancer cells, such as gastric and breast cancer cells $(15,20)$. Our results demonstrated that Res can effectively inhibit the proliferation of LoVo cells, and BMP9 was significantly increased during this process. This evidence implies that BMP9 may be critical for the antiproliferative effect of Res on LoVo cells.

To date, the role of BMP9 in colon cancer remains unknown. Further analysis found that BMP9 was detectable in FHC cells and colon cancer cell lines. However, the level of BMP9 in the FHC cells was distinctly higher than that in the other colon cancer cell lines. These data may also highlight the importance of BMP9 in the regulation of proliferation in colon cancer cells. Our subsequent investigation showed that exog- 
enous expression of BMP9 enhanced the antiproliferation- and apoptosis-inducing effects of Res in LoVo cells, while BMP9 knockdown reduced these effects. Thus, the anticancer activity of Res in LoVo cells may be partly mediated by upregulation of the expression of BMP9. However, how BMP9 mediates this effect remains unknown.

Regarding the canonical BMP/Smad pathway, BMP9 binds to type I receptor (activin receptor-like kinase, including ALK1 and ALK2), followed by Smad1/5/8 phosphorylation (45). However, our data indicated that Res exhibited no substantial effect on the phosphorylation of Smad1/5/8, which implies that the effect of BMP9 on the anticancer activity of Res may not be mediated through the canonical BMP/Smad pathway. Therefore, the non-canonical BMP/Smad pathway, such as p38 MAPK and PI3K/Akt, may be implicated in this effect.

It was previously reported that p38 MAPK is involved in the anticancer effect of Res, and activation of p38 MAPK is also essential for BMP9-induced osteogenesis $(27,41,42)$. Hence, we speculated that the effect of BMP9 on the anticancer activity of Res may be associated with p38 MAPK. Our results showed that Res can apparently increase the phosphorylation of p38 MAPK in a concentration-dependent manner, which is consistent with reports that p38 is involved in the anticancer effect of Res in colon cancer cells $(27,41,42)$. However, how p38 MAPK is activated by Res in colon cancer cells remains unknown. Further assay results showed that exogenous expression of BMP9 enhanced the phosphorylation of p38 MAPK induced by Res, and the p38 MAPK inhibitor (SB203580) decreased the antiproliferative effect of Res, as well as the combination of Res and BMP9. The p38 MAPK inhibitor also reduced the apoptosis induced by Res in the LoVo cells. These data suggest that BMP9 may mediate the anticancer effect of Res partly through activation of p38 MAPK signaling. In fact, p38 MAPK is important not only in regulating the proliferation of cancer cells, but also in mediating the osteogenic differentiation induced by BMP9. The blockage of p38 MAPK greatly reduces BMP9-induced osteogenic differentiation (27). Thus, p38 MAPK should be downstream of BMP9. However, how BMP9 activates p38 MAPK remains unknown. Since Res promotes the activation of $\mathrm{p} 38$ MAPK, upregulates BMP9, and exhibits no substantial effect on Smad1/5/8 phosphorylation, the activation of p38 MAPK by BMP9 may not be mediated through the canonical BMP/Smad pathway. Following treatment with the BMP receptor (BMPR) inhibitor (LDN193189) (46), the Res-induced activation of p38 MAPK was almost abolished, so did the antiproliferative effect of Res in the LoVo cells. Therefore, the activation of BMPR may be necessary for BMP9 to activate p38 MAPK, although Smad1/5/8 is not involved in. These data demonstrated that p38 MAPK may mediate the effect of BMP9 on the anticancer activity of Res in LoVo cells, and BMP9 may activate p38 MAPK through a BMPR-dependent manner.

Taken together, our findings strongly demonstrated that Res is a potent anticancer agent and inhibits the proliferation and promotes the apoptosis of colon cancer cells. The anticancer activity of Res may be mediated by upregulation of BMP9 in colon cancer, by which to activate p38 MAPK in a BMPR-dependent manner. Our findings also indicate that TGF- $\beta$ signaling should be a potential target for colon cancer treatment. However, the exact molecular mechanism of how Res regulates the expression of BMP9 requires further investigation.

\section{Acknowledgements}

We thank Professor Tong-Chuan He of the University of Chicago Medical Center (Chicago, IL, USA) for providing the recombinant adenoviruses. The present study was supported by research grants from the Natural Science Foundation of China (grant nos. NSFC 81372120 and 81572226 to B.-C. H.).

\section{References}

1. Binefa G, Rodríguez-Moranta F, Teule A and Medina-Hayas M: Colorectal cancer: From prevention to personalized medicine. World J Gastroenterol 20: 6786-6808, 2014.

2. Feng QY, Wei Y, Chen JW, Chang WJ, Ye LC, Zhu DX and Xu JM: Anti-EGFR and anti-VEGF agents: Important targeted therapies of colorectal liver metastases. World J Gastroenterol 20: 4263-4275, 2014.

3. Banjerdpongchai R, Chanwikruy Y, Rattanapanone V and Sripanidkulchai B: Induction of apoptosis in the human leukemic U937 cell line by Kaempferia parviflora Wall.ex.Baker extract and effects of paclitaxel and camptothecin. Asian Pac J Cancer Prev 10: 1137-1140, 2009.

4. Yan YX, Li WZ, Huang YQ and Liao WX: The COX-2 inhibitor Celecoxib enhances the sensitivity of KB/VCR oral cancer cell lines to Vincristine by down-regulating P-glycoprotein expression and function. Prostaglandins Other Lipid Mediat 97: 29-35, 2012.

5. Weaver BA: How Taxol/paclitaxel kills cancer cells. Mol Biol Cell 25: 2677-2681, 2014.

6. Das S, Santani DD and Dhalla NS: Experimental evidence for the cardioprotective effects of red wine. Exp Clin Cardiol 12: 5-10, 2007.

7. Thomasset SC, Berry DP, Garcea G, Marczylo T, Steward WP and Gescher AJ: Dietary polyphenolic phytochemicals - promising cancer chemopreventive agents in humans? A review of their clinical properties. Int J Cancer 120: 451-458, 2007.

8. Gehm BD, McAndrews JM, Chien PY and Jameson JL: Resveratrol, a polyphenolic compound found in grapes and wine, is an agonist for the estrogen receptor. Proc Natl Acad Sci USA 94: 14138-14143, 1997.

9. Cottart CH, Nivet-Antoine V and Beaudeux JL: Review of recent data on the metabolism, biological effects, and toxicity of resveratrol in humans. Mol Nutr Food Res 58: 7-21, 2014.

10. Liu YZ, Wu K, Huang J, Liu Y, Wang X, Meng ZJ, Yuan SX, Wang DX, Luo JY, Zuo GW, et al: The PTEN/PI3K/Akt and Wnt/ $\beta$-catenin signaling pathways are involved in the inhibitory effect of resveratrol on human colon cancer cell proliferation. Int J Oncol 45: 104-112, 2014.

11. Chin YT, Hsieh MT, Yang SH, Tsai PW, Wang SH, Wang CC, Lee YS, Cheng GY, HuangFu WC, London D, et al: Antiproliferative and gene expression actions of resveratrol in breast cancer cells in vitro. Oncotarget 5: 12891-12907, 2014.

12. Kai L, Samuel SK and Levenson AS: Resveratrol enhances p53 acetylation and apoptosis in prostate cancer by inhibiting MTA1/ NuRD complex. Int J Cancer 126: 1538-1548, 2010.

13. Sánchez-Duffhues G, Hiepen C, Knaus P and Ten Dijke P: Bone morphogenetic protein signaling in bone homeostasis. Bone 80: 43-59, 2015

14. Chen D, Zhao M, Harris SE and Mi Z: Signal transduction and biological functions of bone morphogenetic proteins. Front Biosci 9: 349-358, 2004.

15. Duan L, Ye L, Wu R, Wang H, Li X, Li H, Yuan S, Zha H, Sun H, Zhang Y, et al: Inactivation of the phosphatidylinositol 3-kinase/ Akt pathway is involved in BMP9-mediated tumor-suppressive effects in gastric cancer cells. J Cell Biochem 116: 1080-1089, 2015.

16. Epstein NE: Basic science and spine literature document bone morphogenetic protein increases cancer risk. Surg Neurol Int 5 (Suppl 15): S552-S560, 2014. 
17. Toofan P, Irvine D, Hopcroft L, Copland $\mathrm{M}$ and Wheadon $\mathrm{H}$ : The role of the bone morphogenetic proteins in leukaemic stem cell persistence. Biochem Soc Trans 42: 809-815, 2014.

18. Kang MH, Oh SC, Lee HJ, Kang HN, Kim JL, Kim JS and Yoo YA: Metastatic function of BMP-2 in gastric cancer cells: The role of PI3K/AKT, MAPK, the NF-кB pathway, and MMP-9 expression. Exp Cell Res 317: 1746-1762, 2011

19. Wang JH, Liu YZ, Yin LJ, Chen L, Huang J, Liu Y, Zhang RX, Zhou LY, Yang QJ, Luo JY, et al: BMP9 and COX-2 form an important regulatory loop in BMP9-induced osteogenic differentiation of mesenchymal stem cells. Bone 57: 311-321, 2013.

20. Wang K, Feng H, Ren W, Sun X, Luo J, Tang M, Zhou L, Weng Y, He TC and Zhang Y: BMP9 inhibits the proliferation and invasiveness of breast cancer cells MDA-MB-231. J Cancer Res Clin Oncol 137: 1687-1696, 2011.

21. Ye L, Kynaston $\mathrm{H}$ and Jiang WG: Bone morphogenetic protein-9 induces apoptosis in prostate cancer cells, the role of prostate apoptosis response-4. Mol Cancer Res 6: 1594-1606, 2008.

22. Luo X, Chen J, Song WX, Tang N, Luo J, Deng ZL, Sharff KA, $\mathrm{He} \mathrm{G}, \mathrm{Bi} \mathrm{Y}, \mathrm{He} \mathrm{BC}$, et al: Osteogenic BMPs promote tumor growth of human osteosarcomas that harbor differentiation defects. Lab Invest 88: 1264-1277, 2008.

23. Herrera B, van Dinther M, Ten Dijke P and Inman GJ: Autocrine bone morphogenetic protein-9 signals through activin receptorlike kinase-2/Smad1/Smad4 to promote ovarian cancer cell proliferation. Cancer Res 69: 9254-9262, 2009.

24. Herrera B, García-Álvaro M, Cruz S, Walsh P, Fernández M, Roncero C, Fabregat I, Sánchez A and Inman GJ: BMP9 is a proliferative and survival factor for human hepatocellular carcinoma cells. PLoS One 8: e69535, 2013.

25. Lv Z, Wang C, Yuan T, Liu Y, Song T, Liu Y, Chen C, Yang M, Tang Z, Shi Q, et al: Bone morphogenetic protein 9 regulates tumor growth of osteosarcoma cells through the Wnt/ $\beta$-catenin pathway. Oncol Rep 31: 989-994, 2014.

26. Huang J, Yuan SX, Wang DX, Wu QX, Wang X, Pi CJ, Zou X, Chen L, Ying LJ, Wu K, et al: The role of COX-2 in mediating the effect of PTEN on BMP9 induced osteogenic differentiation in mouse embryonic fibroblasts. Biomaterials 35: 9649-9659, 2014.

27. Zhao Y, Song T, Wang W, Wang J, He J, Wu N, Tang M, He B and Luo J: P38 and ERK1/2 MAPKs act in opposition to regulate BMP9-induced osteogenic differentiation of mesenchymal progenitor cells. PLoS One 7: e43383, 2012.

28. Cuadrado A and Nebreda AR: Mechanisms and functions of p38 MAPK signalling. Biochem J 429: 403-417, 2010.

29. Luo J, Deng ZL, Luo X, Tang N, Song WX, Chen J, Sharff KA, Luu HH, Haydon RC, Kinzler KW, et al: A protocol for rapid generation of recombinant adenoviruses using the AdEasy system. Nat Protoc 2: 1236-1247, 2007.

30. He TC, Zhou S, da Costa LT, Yu J, Kinzler KW and Vogelstein B: A simplified system for generating recombinant adenoviruses. Proc Natl Acad Sci USA 95: 2509-2514, 1998.

31. He BC, Gao JL, Zhang BQ, Luo Q, Shi Q, Kim SH, Huang E, Gao Y, Yang K, Wagner ER, et al: Tetrandrine inhibits Wnt $/ \beta$ catenin signaling and suppresses tumor growth of human colorectal cancer. Mol Pharmacol 79: 211-219, 2011
32. Chen CS, Ho DR, Chen FY, Chen CR, Ke YD and Su JG: AKT mediates actinomycin D-induced p53 expression. Oncotarget 5: 693-703, 2014.

33. Tárraga López PJ, Albero JS and Rodríguez-Montes JA: Primary and secondary prevention of colorectal cancer. Clin Med Insights Gastroenterol 7: 33-46, 2014.

34. Aggarwal S and Chu E: Current therapies for advanced colorectal cancer. Oncology 19: 589-595, 2005.

35. Wolpin BM and Bass AJ: Managing advanced colorectal cancer: Have we reached the PEAK with current therapies? J Clin Oncol 32: 2200-2202, 2014.

36. Tol $\mathbf{J}$ and Punt CJ: Monoclonal antibodies in the treatment of metastatic colorectal cancer: A review. Clin Ther 32: 437-453, 2010.

37. Khazir J, Riley DL, Pilcher LA, De-Maayer P and Mir BA: Anticancer agents from diverse natural sources. Nat Prod Commun 9: 1655-1669, 2014.

38. Boocock DJ, Faust GE, Patel KR, Schinas AM, Brown VA, Ducharme MP, Booth TD, Crowell JA, Perloff M, Gescher AJ, et al: Phase I dose escalation pharmacokinetic study in healthy volunteers of resveratrol, a potential cancer chemopreventive agent. Cancer Epidemiol Biomarkers Prev 16: 1246-1252, 2007.

39. Vanamala J, Reddivari L, Radhakrishnan S and Tarver C: Resveratrol suppresses IGF-1 induced human colon cancer cell proliferation and elevates apoptosis via suppression of IGF-1R/ Wnt and activation of p53 signaling pathways. BMC Cancer 10: 238,2010

40. Sheth S, Jajoo S, Kaur T, Mukherjea D, Sheehan K, Rybak LP and Ramkumar V: Resveratrol reduces prostate cancer growth and metastasis by inhibiting the Akt/MicroRNA-21 pathway. PLoS One 7: e51655, 2012.

41. Parekh P, Motiwale L, Naik N and Rao KV: Downregulation of cyclin D1 is associated with decreased levels of p38 MAP kinases, Akt/PKB and Pak1 during chemopreventive effects of resveratrol in liver cancer cells. Exp Toxicol Pathol 63: 167-173, 2011.

42. Gweon EJ and Kim SJ: Resveratrol induces MMP-9 and cell migration via the $\mathrm{p} 38$ kinase and PI-3K pathways in HT1080 human fibrosarcoma cells. Oncol Rep 29: 826-834, 2013.

43. Herrera B, Dooley S and Breitkopf-Heinlein K: Potential roles of bone morphogenetic protein (BMP)-9 in human liver diseases. Int J Mol Sci 15: 5199-5220, 2014.

44. Liu Y, Liu Y, Zhang R, Wang X, Huang F, Yan Z, Nie M, Huang J, Wang Y, Wang Y, et al: All-trans retinoic acid modulates bone morphogenic protein 9-induced osteogenesis and adipogenesis of preadipocytes through $\mathrm{BMP} / \mathrm{Smad}$ and $\mathrm{Wnt} / \beta$-catenin signaling pathways. Int J Biochem Cell Biol 47: 47-56, 2014.

45. Luo J, Tang M, Huang J, He BC, Gao JL, Chen L, Zuo GW, Zhang W, Luo Q, Shi Q, et al: TGFbeta/BMP type I receptors ALK1 and ALK2 are essential for BMP9-induced osteogenic signaling in mesenchymal stem cells. J Biol Chem 285: 29588-29598, 2010.

46. Sanvitale CE, Kerr G, Chaikuad A, Ramel MC, Mohedas AH, Reichert S, Wang Y, Triffitt JT, Cuny GD, Yu PB, et al: A new class of small molecule inhibitor of BMP signaling. PLoS One 8: e62721, 2013. 